\title{
Fase Preliminar de un Proyecto de Investigación
}

\author{
Francisca Gil Latorre ${ }^{a}$
}

\begin{abstract}
a Unidad Docente.
Medicina de Familia y Comunitaria de Valencia, España.
\end{abstract}

Correspondencia:

Francisca Gil Latorre, C/ Juan de Garay n²1, 46017 - Valencia, España. Telf.: 686323261, e-mail: gil_fra@gva.es.

Recibido el 21 de enero de 2010.

Aceptado para su publicación el 28 de enero de 2010.

\section{RESUMEN}

La Pregunta de Investigación, los Antecedentes y Justificación del tema, la Hipótesis y los Objetivos forman parte de la fase preliminar del proyecto de investigación.

La pregunta de investigación debe ser formulada de forma precisa y clara. Sin una buena pregunta de investigación no se puede llevar a cabo un estudio de investigación. La pregunta debe sugerir la dirección que ha de tomar la investigación y orientar sobre la metodología a seguir en el diseño y la ejecución del estudio.

Una vez que hemos detectado un problema deberemos realizar un análisis del conocimiento sobre el tema, para lo cual es fundamental una revisión bibliográfica, y preguntarse si la realización del estudio está justificada.

La elaboración de los objetivos del estudio va encaminada a responder la pregunta de investigación.

Palabras clave: Investigación, Atención Primaria de Salud.

\section{ABSTRACT}

\section{Preliminary stage of a Research Project}

The Research Question, Background, Rationale, Hypothesis and Objectives comprise the preliminary stage of a research project.

The research question should be precise and clear. Without a strong research question you cannot conduct a research study. The question suggests the direction the research should take and guides the methodology, design and execution of the study.

Once you have found a problem to be approached, the knowledge on the topic should be analysed. To this end a complete review of the literature is essential. You must also ask yourself if the performance of the study can be justified.

The study objectives will be directed towards answering the research question.

Key words: Research, Primary Health Care.

\section{INTRODUCCIÓN}

El ser humano, casi desde siempre, ha estado preocupado en entender la naturaleza de los fenómenos que se presentan a sus sentidos. El medio a través del cual esta comprensión se puede llevar a cabo es: por la experiencia, el razonamiento y la investigación, existiendo una complementariedad entre ellos.

Tanto el razonamiento como la experiencia son elementos fundamentales en el planteamiento de preguntas de investigación y generación de hipótesis, aunque limitadas en la obtención de información "objetiva".

Se define INVESTIGACIÓN como un proceso sistemático, organizado y objetivo, destinado a responder a una pregunta.

Es Sistemático porque se realiza siguiendo un orden y una metodología preestablecida.

Es Organizado porque se entiende que todos los miembros de un equipo conocen a la perfección lo que hay que hacer durante todo el estudio, apli- 
cando las mismas definiciones y criterios a todos los participantes y actuando de forma idéntica ante cualquier duda. Para conseguirlo, es imprescindible escribir un protocolo donde se especifiquen todos los detalles relacionados con el estudio.

Es Objetivo porque las conclusiones que se obtienen no se basan en impresiones subjetivas, sino en hechos que se han observado y medido, y en su interpretación se evita cualquier prejuicio que los responsables del estudio pudieran tener. Por lo que Investigar es diseñar y poner en marcha procesos activos que proporcionan resultados analizables e interpretables, que aporten nuevos datos, desconocidos o no demostrables previamente.

Cuando se habla de la necesidad de investigar la pregunta que surge es: ¿para qué investigar?, ¿para qué desarrollar un proyecto de investigación?

Saber contestar a la pregunta de ¿para qué? evalúa la pertinencia de la investigación, y la pertinencia de un estudio está íntimamente relacionada con los resultados esperados y su generalización. También hay que contestar a la pregunta ¿cómo?, es decir, el método. La delimitación del problema determina el alcance de las conclusiones donde se insertan todas las interrogantes de investigación.

Para el trabajo en Atención Primaria (AP), debemos adquirir una serie de conocimientos específicos cuya práctica se justifica en función de un cuerpo de conocimientos teóricos. Por medio de la investigación desarrollamos este cuerpo de conocimientos, y el método empleado para ello es el método científico. El método científico se define como el estudio empírico controlado, crítico y sistemático de hipótesis que intentan explicar presuntas relaciones entre varios fenómenos (Kerlinger, 1973).

¿Qué actividades realiza un equipo investigador?: medir fenómenos, comparar los resultados con los obtenidos en la población a la que se quieren referir e interpretarlos en función de los conocimientos actuales y teniendo en cuenta las variables que puedan haber influido en estos resultados. Estas actividades han de orientarse a la resolución de problemas concretos utilizando una metodología adecuada, el método científico.

\section{¿QUÉ SE INVESTIGA EN ATENCIÓN PRIMARIA?}

La AP contempla la historia natural de la enfermedad en todo su aspecto: contempla el ciclo vital humano continuamente, y nadie está en mejor disposición para observar desde los antecedentes familiares hasta las consecuencias últimas de cualquier problema de salud. Este es el primer justificante de la investigación en AP, sea desde la perspectiva clíni- ca, epidemiológica u organizativa, o desde la evaluación de las intervenciones del médico de familia sobre la salud del individuo, familia y comunidad.

Los temas mas frecuentes que se investigan en AP suelen ser:

Historia natural de las enfermedades comunes en la población y niveles apropiados de atención para esos problemas.

Efectividad de la atención: de estrategias preventivas, diagnósticas y terapéuticas.

Mediadores no médicos de resultados de salud: impacto de la familia, etnia, influencias sociales, relación médico-paciente.

Poblaciones desfavorecidas: áreas rurales, interior de grandes ciudades, ancianos, madres y niños, minorías étnicas, SIDA, minusválidos, discapacitados, pacientes que dependen crónicamente de la atención médica.

Investigación orientada a la práctica clínica: comportamientos, decisiones clínicas, derivación de pacientes, tipo de consultas, pautas de práctica médica, tecnología apropiada.

Diseminación de protocolos: pautas de práctica médica, tecnología apropiada.

Investigación sobre métodos: codificación y clasificación de problemas de salud, medidas de estados funcionales, medidas de resultados de episodios de atención, satisfacción de los pacientes, metaanálisis, investigaciones cuasi-experimentales, cualitativas, participativas.

\section{Modos alternativos de organización de la con- sulta.}

Estudios de la eficacia y de la calidad de la asistencia: hospitalizaciones innecesarias.

Estudios de utilización de fármacos: prescripción innecesaria de antibióticos.

Investigación sobre la asistencia urgente desde la perspectiva de la AP.

Efectividad de intervenciones: medidas higiénicodietéticas en diabéticos, hipertensos, dislipémicos y obesos.

Evaluación de los resultados: de las iniciativas de mejora de la calidad.

\section{¿A QUÉ SE LLAMA DISEÑO DE UN PROYECTO DE INVESTIGACIÓN?}

El diseño de un proyecto de investigación es un documento que recoge todos los procedimientos, mé- 
todos y técnicas mediante los cuales el investigador selecciona los individuos, recoge la información y analiza los resultados.

Investigar no es simplemente almacenar y analizar información de manera indiscriminada, sino que debe ser aplicada a la resolución de problemas concretos utilizando la metodología basada en el método científico.

Parte de la identificación de un problema, cuya respuesta tiene en consideración el conocimiento sobre el mismo en el momento de planificar la investigación. La hipótesis conceptual generada del problema, e íntimamente relacionada con la hipótesis operativa, dará luz a la recogida de datos, cuya información útil vendrá dada por el análisis e interpretación de los mismos, destinados a responder a una pregunta. En el Anexo 1 se presenta un esquema de la fase de planificación de un proyecto de investigación.

Vamos a comentar la fase preliminar de la planificación de un proyecto de investigación:

\section{Identificación de un Problema}

Un problema de investigación es una falta de certeza sobre algún hecho o fenómeno que el investigador desea responder y cuyo planteamiento está en relación con la capacidad del investigador para observar y generar ideas.

La formulación de una pregunta de investigación es un paso, el más importante, en el proceso de diseño y desarrollo de una investigación.

¿Cómo generar ideas para iniciar la búsqueda de una pregunta? y ¿cómo iniciar la búsqueda de una pregunta?

La generación de ideas puede surgir tanto de la participación en sesiones bibliográficas y congresos como de la observación cuidadosa de pacientes, de la experiencia previa del investigador en anteriores estudios o en otros estudios, de la docencia, etc.

Proceso que se lleva a cabo en una pregunta de Investigación a partir de un problema en la consulta

\section{Caso clínico:}

Maria acude a la consulta con dolor en las articulaciones y rigidez matutina, diagnosticada hace 20 años de osteoartritis. Este dolor es tan incapacitante que le impide llevar a cabo su vida diaria, ni tan siquiera mover las manos (unos ejercicios que le enseñaron no los puede realizar). Ya no sabe qué fármacos tomar y esto le lleva a estar con cambios de humor, dice que no puede más. Los medicamen- tos que toma no le sientan bien y a veces no se los toma. Dice que a veces le calma y le relaja cuando escucha un CD de música clásica.

El dolor crónico es una de las causas mas frecuentes en pacientes adultos atendidos en las consultas de AP. El $50-70 \%$ están parcial o totalmente incapacitados y el resto, el $30 \%$, lo están permanentemente días, semanas o meses.

\section{Marco teórico:}

En estudios previos se ha demostrado que la música produce numerosos efectos beneficiosos en el ser humano. Acentúa el desarrollo emocional y mejora las percepciones, estimulando nuestros receptores sensoriales, la motricidad y la afectividad de las personas. Actúa sobre el sistema nervioso central provocando efectos estimulantes y sedantes, disminuye la ansiedad y el estrés. Asocia reacciones de RELAJACIÓN MUSCULAR con una determinada música. Como la percepción del dolor modula la ansiedad y el estrés, algún grado de RELAJACIÓN puede ayudar a reducirlo. Con todo esto nos queda el interrogante: si el efecto de la musicoterapia es beneficioso en el dolor crónico osteomuscular, y puede disminuir el escalón terapéutico o estabilizar el dolor en el escalón actual, y además si se puede determinar el número de sesiones necesarias para obtener resultados.

\section{¿Cómo se formula la pregunta de investigación?}

¿Cómo son los pacientes?, ¿hay o no Intervención?, ¿compararemos con otra intervención?, ¿Cómo evaluaremos el resultado? Se trata, mediante una experiencia empírica sistemática, de aportar una "respuesta", una solución satisfactoria al problema. A ser posible, el problema debería ser planteado en forma de pregunta.

\section{Características de una pregunta de investigación}

Factibilidad: se debe conocer, desde el inicio, los límites y problemas que surgirán al estudiar un problema determinado, antes de invertir tiempo y esfuerzo en líneas en las que luego no se podrá trabajar. La factibilidad vendrá determinada por:

Número de individuos: hay que hacer un cálculo del tamaño de la muestra, teniendo en cuenta las características del estudio y ver si contamos con el número de dichos individuos. El investigador debe estar seguro de que habrá suficientes individuos que participen en el estudio.

Experiencia técnica: los investigadores deben poseer la técnica y la experiencia necesarias para llevar acabo el estudio. 
Coste en tiempo y dinero: hay que estimar los costes y el tiempo que se llevará cada parte del proyecto.

Alcance: hay que concentrarse en los objetivos del estudio e intentar responderlos, y no querer contestar otras preguntas secundarias.

Interesante: para el investigador en cuanto al conocimiento del tema, beneficio económico y curricular.

\section{Novedosa: si aporta nueva información.}

Ética: Si el estudio supone riesgo para los individuos, se deberán buscar otras formas de contestar a la pregunta. Si se duda, se recurrirá a los comités de ética.

Relevante: para el conocimiento científico, para la política clínica y sanitaria y para líneas de investigación futuras.

En nuestro caso formularíamos la pregunta de la siguiente forma: ¿en pacientes con dolor crónico osteomuscular, atendidos en atención primaria, es efectiva la musicoterapia en el alivio del dolor crónico osteomuscular?

\section{ANTECEDENTES Y JUSTIFICACIÓN DEL TEMA}

Una vez que hemos detectado un problema deberemos realizar un análisis del conocimiento sobre el tema, para lo cual es fundamental una revisión bibliográfica, y preguntarse si la realización del estudio está justificada (pertinencia), y si es viable.

\section{HIPÓTESIS}

Las hipótesis sintetizan la pregunta científica a la que pretende dar respuesta la investigación y permiten enjuiciar parcialmente la originalidad, la factibilidad, la relevancia científica y socio-sanitaria del proyecto.

La elaboración de los objetivos del estudio, encaminada a responder la pregunta de investigación, requiere un trabajo detenido, ya que muchos aspectos del diseño dependen de lo que se enuncie como objetivos.

Lo más adecuado es plantear un estudio para contestar a una ÚNICA PREGUNTA PRINCIPAL que contemple, en todo caso, respuesta a otras preguntas secundarias o complementarias a la principal. La razón estriba en la complejidad del diseño de un estudio que se enfoca en responder más de una pregunta principal.

La hipótesis se expresa como una "verdad provisional" relacionada con el marco teórico e indica en qué forma la refutación de la hipótesis hará avanzar el conocimiento en el campo específico. Las hipó- tesis son típicas de los estudios analíticos, siendo innecesarias en los estudios descriptivos.

En nuestro estudio formularíamos la hipótesis de la siguiente forma: "el efecto de la musicoterapia es beneficioso en el alivio del dolor crónico de origen osteomuscular en pacientes atendidos en Atención Primaria".

\section{OBJETIVOS}

Hay que expresar un objetivo en términos generales (objetivo general), seguido de uno o varios objetivos específicos. En la formulación de los objetivos generales se especifica el fenómeno, suceso o intervención que quieren estudiar y en qué población. Ningún estudio de investigación puede tener una validez correcta si previamente a la recogida de datos no se han especificado los objetivos.

En nuestro estudio formularíamos el objetivo general de la siguiente forma: "Valorar el umbral del dolor y la calidad de vida de pacientes con dolor crónico osteomuscular y usuarios del Centro de Salud sometidos a musicoterapia, según la Escala Analgésica del dolor de la OMS, mediante aplicación de las láminas COOP WONCA adaptadas al español y el Cuestionario del Dolor Español".

A medida que vayamos desarrollando las fases más operativas de la planificación del estudio, tales como la elección de la población o sujetos sobre los que vamos a realizar las mediciones, y las variables que vamos a medir para describir y explicar los fenómenos de interés, podremos enunciar objetivos más específicos.

El Objetivo Específico de un estudio "ANALÍTICO" consiste en estudiar la relación entre algún factor y CRITERIO DE EVALUACIÓN O VARIABLE RESPUESTA, en una población definida.

Los componentes de un objetivo "ANALÍTICO" son: Factor de estudio: que corresponde a la exposición o intervención de interés.

Variable respuesta: con la que se pretende medir el efecto o la asociación.

Población a estudio: conjunto de sujetos en los que se realizan las mediciones.

Período temporal: espacio de tiempo en el que se recogen las observaciones.

EL Objetivo Específico de un estudio "DESCRIPTIVO" suele ser fácil de formular, va orientado a investigar las características de un grupo concreto de población.

Consta de los siguientes componentes:

Factor de estudio: corresponde a la característica del estudio. 
Medida de frecuencia.

Población de estudio: conjunto de sujetos en los que se realizan las mediciones.

Período de tiempo: momento en el que se recogen las observaciones.

Tanto los Objetivos generales como los específicos se formulan con el verbo en infinitivo.

En nuestro estudio formularíamos los distintos objetivos específicos de la siguiente forma:

1."Describir el nivel de calidad de vida de la población de estudio en tratamiento con aspirina, paracetamol, AINES, o pirazolonas, mediante las laminas COOP-WONCA, adaptado al español, antes y después de aplicar la intervención musicoterapia, 20 minutos al día durante dos semanas".

2."Describir el nivel de calidad de vida de la población de estudio en tratamiento con opióides débiles (tramadol, codeína, dihidrocodeina), mediante las laminas COOP-WONCA, adaptado al español, antes y después de aplicar la intervención musicoterapia".

3."Describir el nivel de calidad de vida de la población de estudio en tratamiento con opióides potentes (morfina, fentanilo, TTS), mediante las laminas COOP-WONCA, adaptado al español, antes y después de aplicar la intervención musicoterapia".

4."Describir la calidad de vida de la población de estudio, no sometida a tratamiento analgésico o que no este incluida en ninguno de los apartados anteriores, mediante las laminas COOP-WONCA, adaptado al español, antes y después de aplicar la intervención musicoterapia".

\section{CONCLUSIONES}

El desarrollo del proyecto comienza con la idea general o particular sobre un determinado aspecto de la práctica clínica, diagnóstico, tratamiento, actuaciones, etc. La idea terminará de concretarse tras la revisión bibliográfica del tema, necesaria para situar el tema a investigar en el conocimiento actual. La Elaboración de un Proyecto va a requerir tiempo y varias revisiones del mismo. Únicamente un buen diseño carente de errores, que intente responder una pregunta de interés, merecerá todo el esfuerzo ulterior de su puesta en marcha, recogida y análisis de resultados.

Lo que define a un proyecto de investigación son los objetivos que pretende alcanzar. La definición clara de objetivos es el primer paso en la decisión del diseño a utilizar, las variables y el tipo de análisis.

\section{BIBLIOGRAFÍA}

- Albert Ros X, Gil Latorre F, Gutiérrez Sigler D. CICAP Investigación Clínica en Atención Primaria. Madrid: Luzán; 2004. p. 1-326.

- Argimón JM, Jiménez J. Métodos de investigación. Madrid: Ed. Doyma; 1996.

- Coughlin SS, Beauchamp LT. Ethics, scientific validity and the design of epidemiologic studies. Epidemiology. 1992; 3:343-47.

- Boch F. Clínica del dolor. Proyección en el sistema nacional de salud en Cuba. Conferencia ofrecida en taller internacional: Dolor tratamiento integral. XXII congreso latinoamericano de anestesiología. La Habana; 1995.

- Ferral BA. Pain management in elderly people. J Am Geriratr Soc. $1991 ; 39: 64-73$

Anexo 1. FASES DEL PROYECTO DE INVESTIGACIÓN

FASE PRELIMINAR:

1. Identificación de un problema.

2. Antecedentes y justificación del tema

3. Hipótesis u Objetivos.

ELECCIÓN DE UN DISEÑO:

4. Tipo de estudio.

PLANIFICACIÓN:

5. Ámbito:

- Población diana, población de estudio y muestra.

- Criterios de selección.

- Tamaño de la muestra.

- Sistema de muestreo.

- Asignación a grupos de estudio.

6. Variables:

- Selección, definición y escala de medida

7. Recogida de datos:

- Instrumentos de medida.

- Organización del trabajo de campo.

- Calidad de los datos.

8. Estrategia de análisis.

9. Dificultades y limitaciones.

10. Organización:

- Cronograma.

- Aspectos éticos.

- Presupuestos. 